\title{
AN EMBEDDING OF SCHWARTZ DISTRIBUTIONS IN THE ALGEBRA OF ASYMPTOTIC FUNCTIONS
}

\author{
MICHAEL OBERGUGGENBERGER \\ Institut furr Mathematik und Geometrie \\ Universität Innsbruck \\ A-6020 Inssbruck, AUSTRIA \\ michael@matl.uibk.ac.at \\ and \\ TODOR TODOROV \\ Mathematics Department \\ California Polytechnic State University \\ San Luis Obispo, California 93407, USA \\ ttodorov@oboe.calpoly.edu
}

(Received October 28, 1996 and in revised form June 21, 1997)

\begin{abstract}
We present a solution of the problem of multiplication of Schwartz distributions by embedding the space of distributions into a differential algebra of generalized functions, called in the paper "asymptotic function," similar to but different from J. F Colombeau's algebras of new generalized functions.
\end{abstract}

KEY WORDS AND PHRASES: Schwartz distributions, nonlinear theory of generalized functions, asymptotic expansion, nonstandard analysis, nonstandard asymptotic analysis

1991 AMS SUBJECT CLASSIFICATION CODES: 03H05, 12J25, 46F10, 46S20

\section{INTRODUCTION}

The main purpose of this paper is to prove the existence of an embedding $\Sigma_{D, \Omega}$ of the space of Schwartz distributions $\mathcal{D}^{\prime}(\Omega)$ into the algebra of asymptotic functions ${ }^{\rho} E(\Omega)$ which preserves all linear operations in $\mathcal{D}^{\prime}(\Omega)$. Thus, we offer a solution of the problem of multiplication of Schwartz distributions since the multiplication within $\mathcal{D}^{\prime}(\Omega)$ is impossible (L. Schwartz [1]).

The algebra ${ }^{\rho} E(\Omega)$ is defined in the paper as a factor space of nonstandard smooth functions The field of the scalars ${ }^{\rho} \mathbb{C}$ of the algebra ${ }^{\rho} E(\Omega)$, coincides with the complex counterpart of A. Robinson's asymptotic numbers-known also as "Robinson's field with valuation" (see A Robinson [2]) and A. H. Lightstone and A. Robinson [3]). The embedding $\Sigma_{D, \Omega}$ is constructed in the form $\Sigma_{D, \Omega}=Q_{\Omega} \circ D * \Pi \bullet^{*}$ where (in backward order): * is the extension mapping (in the sense of nonstandard analysis), - is the Schwartz multiplication in $\mathcal{D}^{\prime}(\Omega)$ (more precisely, its nonstandard extension in ${ }^{*} \mathcal{D}^{\prime}(\Omega)$ ), * is the convolution operator (more precisely, its nonstandard extension), 。 denotes "composition," $Q_{\Omega}$ is the quotient mapping (in the definition of the algebra of asymptotic functions) and $D$ and $\Pi_{\Omega}$ are fixed nonstandard internal functions with special properties whose existence is proved in this paper.

Our interest in the algebra ${ }^{\rho} E(\Omega)$ and the embedding $\mathcal{D}^{\prime}(\Omega) \subset{ }^{\rho} E(\Omega)$, is due to their role in the problem of multiplication of Schwartz distributions, the nonlinear theory of generalized functions and its applications to partial differential equations (M. Oberguggenberger [4]), (T. Todorov [5] and [6]). In particular, there is a strong similarity between the algebra of asymptotic functions ${ }^{\rho} E(\Omega)$ and its generalized scalars ${ }^{\rho} \mathbb{C}$, discussed in this paper, and the algebra of generalized functions $\mathcal{G}(\Omega)$ and their 
generalized scalars $\overline{\mathbb{C}}$, introduced by J. F. Colombeau in the framework of standard analysis (J. F. Colombeau [7], pp. 63, 138 and J. F. Colombeau [8], §8.3, pp. 161-166). We should mention that the involvement of nonstandard analysis has resulted in some improvements of the corresponding standard counterparts; one of them is that $P \mathbb{C}$ is an algebraically closed field while its standard counterpart $\overline{\mathbb{C}}$ in J. F. Colombeau's theory is a ring with zero divisors.

This paper is a generalization of some results in [9] and [10] (by the authors of this paper, respectively) where only the embedding of the tempered distributions $\mathcal{S}^{\prime}\left(\mathbb{R}^{d}\right)$ in ${ }^{\rho} E\left(\mathbb{R}^{d}\right)$ has been established. The embedding of all distributions $\mathcal{D}^{\prime}(\Omega)$, discussed in this paper, presents an essentially different situation. We should mention that the algebra ${ }^{\rho} E\left(\mathbb{R}^{d}\right)$ was recently studied by $R$. F. Hoskins and J. Sousa Pinto [11].

Here $\Omega$ denotes an open set of $\mathbb{R}^{d}$ ( $d$ is a natural number), $E(\Omega)=C^{\infty}(\Omega)$ and $\mathcal{D}(\Omega)=C_{0}^{\infty}(\Omega)$ denote the usual classes of $C^{\infty}$-functions on $\Omega$ and $C^{\infty}$-functions with compact support in $\Omega$ and $\mathcal{D}^{\prime}(\Omega)$, and $E^{\prime}(\Omega)$ denote the classes of Schwartz distributions on $\Omega$ and Schwartz distributions with compact support in $\Omega$, respectively. As usual, $\mathbb{N}, \mathbb{R}, \mathbf{R}_{+}$and $\mathbb{C}$ will be the systems of the natural, real, positive real and complex numbers, respectively, and we use also the notation $\mathbb{N}_{0}=\{0\} \cup \mathbb{N}$. For the partial derivatives we write $\partial^{\alpha}, \alpha \in \mathbb{N}_{0}^{d}$. If $\alpha=\left(\alpha_{1}, \ldots, \alpha_{d}\right)$ for some $\alpha \in \mathbb{N}_{0}^{d}$, then we write $|\alpha|=\alpha_{1}+\ldots+\alpha_{d}$ and if $x=\left(x_{1}, \ldots, x_{d}\right)$ for some $x \in \mathbb{R}^{d}$, then we write $x^{\alpha}=x_{1}^{\alpha_{1}} x_{2}^{\alpha_{2}} \ldots x_{d}^{\alpha_{2}} \ldots x_{d}^{\alpha_{d}}$. For a general reference to distribution theory we refer to $H$. Bremermann [12] and V. Vladimirov [13].

Our framework is a nonstandard model of the complex numbers $\mathbb{C}$, with degree of saturation larger than $\operatorname{card}(\mathbb{N})$. We denote by ${ }^{*} \mathbb{R},{ }^{*} \mathbb{R}_{+},{ }^{*} \mathbb{C},{ }^{*} E(\Omega)$ and ${ }^{*} \mathcal{D}(\Omega)$ the nonstandard extensions of $\mathbb{R}, \mathbb{R}_{+}, \mathbb{C}$, $E(\Omega)$ and $\mathcal{D}(\Omega)$, respectively. If $X$ is a set of complex numbers or a set of (standard) functions, then ${ }^{*} X$ will be its nonstandard extension and if $f: X \rightarrow Y$ is a (standard) mapping, then * $f:{ }^{*} X \rightarrow{ }^{*} Y$ will be its nonstandard extension. For integration in " $\mathbb{R}^{d}$ we use the $*$-Lebesgue integral. We shall often use the same notation, $\|x\|$, for the Euclidean norm in $\mathbb{R}^{d}$ and its nonstandard extension in ${ }^{*} \mathbb{R}^{d}$. For a short introduction to nonstandard analysis we refer to the Appendix in T. Todorov [6]. For a more detailed exposition we recommend $T$. Lindstrom [14], where the reader will find many references to the subject.

\section{TEST FUNCTIONS AND THEIR MOMENTS}

In this section we study some properties of the test functions in $\mathcal{D}\left(\mathbf{R}^{d}\right)$ (in a standard setting) which we shall use subsequently.

Following (J.F. Colombeau [7], p. 55), for any $k \in \mathbb{N}$ we define the set of test functions:

$$
\begin{aligned}
A_{k}=\left\{\varphi \in \mathcal{D}\left(\mathbb{R}^{d}\right): \varphi \text { is real-valued, } \varphi(x)=0 \text { for }\|x\| \geq 1 ;\right. \\
\left.\qquad \int_{\mathbb{R}^{d}} \varphi(x) d x=1 \text { and } \int_{\mathbb{R}^{d}} x^{\alpha} \varphi(x) d x=0 \text { for } \alpha \in \mathbb{N}_{0}^{d}, \quad 1 \leq|\alpha| \leq k\right\} .
\end{aligned}
$$

Obviously, $A_{1} \supset A_{2} \supset A_{3} \supset \ldots$ Also, we have $A_{k} \neq \emptyset$ for all $k \in \mathbb{N}$ (J.F. Colombeau [7], Lemma (3.3.1), p. 55).

In addition to the above we have the following result:

LEMMA 2.2. For any $k \in \mathbb{N}$

$$
\inf _{\varphi \in A_{k}}\left(\int_{\mathbf{R}^{d}}|\varphi(x)| d x\right)=1
$$

More precisely, for any positive real $\delta$ there exists $\varphi$ in $A_{k}$ such that

$$
1 \leq \int_{\mathbb{R}^{d}}|\varphi(x)| d x \leq 1+\delta .
$$

In addition, $\varphi$ can be chosen symmetric. 
PROOF. We consider the one dimensional case $d=1$ first. Start with some fixed positive (real valued) $\psi$ in $\mathcal{D}(\mathbb{R})$ such that $\psi(x)=0$ for $|x| \geq 1$ and $\int_{\mathbb{R}} \psi(x) d x=1$ ( $\psi$ can be also chosen symmetric if needed). We shall look for $\varphi$ in the form:

$$
\varphi(x)=\sum_{j=0}^{k} c_{j} \psi\left(\frac{x}{\epsilon^{j}}\right)
$$

$x \in \mathbb{R}, \epsilon \in \mathbb{R}_{+}$. We have to find $c$, for which $\varphi \in A_{k}$. Observing that

$$
\int_{\mathbb{R}} x^{i} \psi\left(\frac{x}{\epsilon^{j}}\right) d x=\epsilon^{(i+1) j} \int_{\mathbb{R}} y^{i} \psi(y) d y
$$

for $i=0,1, \ldots, k$, we derive the system for linear equations for $c_{j}$ :

$$
\left\{\begin{array}{l}
\sum_{j=0}^{k} c_{j} \epsilon^{\jmath}=1, \\
\left(\int_{\mathbb{R}} y^{i} \psi(y) d y\right) \sum_{j=0}^{k} c_{j} \epsilon^{(i+1) \jmath}=0, \quad i=1, \ldots, k .
\end{array}\right.
$$

The system is certainly satisfied, if

$$
\left\{\begin{array}{l}
\sum_{j=0}^{k} c_{j} \epsilon^{j}=1, \\
\sum_{j=0}^{k} c_{j} \epsilon^{(1+1) \jmath}=0, \quad i=1, \ldots, k,
\end{array}\right.
$$

which can be written in the matrix form $V_{k+1}(\epsilon) C=B$, where $V_{k+1}(\epsilon)$ is Vandermonde $(k+1) \times(k+1)$ matrix, $C$ is the column of the unknowns $c$, and $B$ is the column whose top entry is 1 and all others are 0 . For the determinant we have $\operatorname{det} V_{k+1}(\epsilon) \neq 0$ for $\epsilon \neq 1$, therefore, the system has a unique solution $\left(c_{1}, c_{1}, c_{2}, \ldots, c_{k}\right)$. Our next goal is to show that this solution is of the form:

$$
c_{\jmath}= \pm \frac{\epsilon^{\alpha},\left(1+\epsilon P_{j}(\epsilon)\right)}{\epsilon^{\beta}(1+\epsilon P(\epsilon))}
$$

where $P_{j}$ and $P$ are polynomials and

$$
\alpha_{\jmath}=\sum_{q=1}^{k-1} q(k+1-q)+\sum_{m=j}^{k-1}(k+1-m)
$$

for $0 \leq j \leq k$, and

$$
\beta=k+\sum_{q=1}^{k-1} q(k+1-q) .
$$

The coefficients $c_{0}, c_{1}, \ldots, c_{k}$ will be found by Cramer's rule. The formula for Vandermonde determinants gives

$$
\begin{aligned}
\prod_{m=1}^{k} \prod_{q=m+1}^{k+1}\left(\epsilon^{q}-\epsilon^{m}\right) & =\prod_{m=1}^{k}\left(\prod_{q=m+1}^{k+1} \epsilon^{m}\left(\epsilon^{q-m}-1\right)\right) \\
& =\epsilon^{\beta} \prod_{m=1}^{k} \prod_{q=m+1}^{k+1}\left(\epsilon^{q-m}-1\right)= \pm \epsilon^{\beta}(1+\epsilon P(\epsilon))
\end{aligned}
$$

for some polynomial $P$, where 


$$
\beta=\sum_{m=1}^{k} m(k+1-m)=k+\sum_{m=1}^{k-1} m(k+1-m) .
$$

To calculate the numerator in (2.3), we have to replace the $j$ th column of the matrix by the column $B$ (whose top entry is 1 and all others are 0 ) and calculate the resulting determinant $D_{\jmath}$. Consider first the case $1 \leq j \leq k-1$. By developing with respect to the $j$ th column, we get

$$
D_{\jmath}= \pm \operatorname{det}\left(\begin{array}{ccccccc}
1, & \epsilon^{2}, & \ldots & \epsilon^{2(j-1)}, & \epsilon^{2(\jmath+1)}, & \ldots & \epsilon^{2 k} \\
1, & \epsilon^{3}, & \ldots & \epsilon^{3(j-1),} & \epsilon^{3(\jmath+1)}, & \ldots & \epsilon^{3 k} \\
\ldots & \ldots & \ldots & \ldots & \ldots & \ldots & \ldots \\
1, & \epsilon^{k+1}, & \ldots & \epsilon^{(k+1)(\jmath-1)}, & \epsilon^{(k+1)(\jmath+1)}, & \ldots & \epsilon^{(k+1) k}
\end{array}\right) .
$$

We factor out $\epsilon^{2}, \epsilon^{4}, \ldots, \epsilon^{2(\jmath-1)}, \epsilon^{2(\jmath+1)}, \ldots, \epsilon^{2 k}$ and obtain:

$$
\begin{aligned}
D_{\jmath}= \pm \epsilon^{1 \cdot 2} \epsilon^{2 \cdot 2} \ldots \epsilon^{(\jmath-1) \cdot 2} \epsilon^{(j+1) \cdot 2} \ldots \epsilon^{2 k} \\
\quad \times \operatorname{det}\left(\begin{array}{cccccccc}
1, & 1, & 1, & \ldots & 1, & 1, & \ldots & 1 \\
1, & \epsilon, & \epsilon^{2}, & \ldots & \epsilon^{(\jmath-1)}, & \epsilon^{(\jmath+1)}, & \ldots & \epsilon^{k} \\
\ldots & \ldots & \ldots & \ldots & \ldots & \ldots & \ldots & \ldots \\
1, & \epsilon^{k-1}, & \epsilon^{2(k-1)}, & \ldots & \epsilon^{(j-1)(k-1)}, & \epsilon^{(j+1)(k-1)}, & \ldots & \epsilon^{k(k-1)}
\end{array}\right) .
\end{aligned}
$$

The latter is a Vandermonde determinant again, and we have

$$
\begin{aligned}
D_{j}= & \pm \epsilon^{1 \cdot 2+2 \cdot 2+\ldots+(j-1) 2+(\jmath+1) 2+\ldots k \cdot 2} \\
& \times(\epsilon-1)\left(\epsilon^{2}-1\right)\left(\epsilon^{3}-1\right) \ldots\left(\epsilon^{j-1}-1\right)\left(\epsilon^{j+1}-1\right) \ldots\left(\epsilon^{k}-1\right) \\
& \times\left(\epsilon^{2}-\epsilon\right)\left(\epsilon^{3}-\epsilon\right) \ldots\left(\epsilon^{j-1}-\epsilon\right)\left(\epsilon^{j+1}-\epsilon\right) \ldots\left(\epsilon^{k}-\epsilon\right) \\
& \times \ldots \ldots \ldots \ldots \ldots \ldots \ldots \ldots \ldots \ldots \ldots \ldots \ldots \ldots \ldots \ldots \ldots \ldots \ldots \ldots \ldots \ldots \ldots \ldots \ldots \ldots \ldots \ldots \ldots \ldots \ldots \ldots \ldots \ldots \ldots \\
& \left(\epsilon^{j-1}-\epsilon^{j-2}\right)\left(\epsilon^{j+1}-\epsilon^{j-2}\right) \ldots\left(\epsilon^{k}-\epsilon^{j-2}\right) \\
& \times\left(\epsilon^{j+1}-\epsilon^{j-1}\right) \ldots\left(\epsilon^{k}-\epsilon^{j-1}\right) \\
& \ldots \ldots \ldots \ldots \ldots \ldots \ldots \ldots \ldots
\end{aligned}
$$

Hence, factoring out $\epsilon^{(i-1)(k-\imath)}$ in the ith row above, we get $D_{\jmath}= \pm \epsilon^{\alpha}\left(1+\epsilon P_{j}(\epsilon)\right)$ for some polynomials $P_{j}(\epsilon)$ and

$$
\begin{aligned}
\alpha_{j}= & 1 \cdot 2+2 \cdot 2+\ldots+(j-1) \cdot 2+(j+1) \cdot 2+\ldots+k \cdot 2 \\
& +1 \cdot(k-2)+2(k-3)+\ldots+(j-1)(k-j) \\
& +(j+1)(k-j-1)+\ldots+(k-1) \cdot 1 \\
= & 1 \cdot k+2(k-1)+\ldots+(j-1)(k-j+2)+(j+1)(k-j+1)+\ldots+(k-1) \cdot 3+k \cdot 2 \\
= & \sum_{q=1}^{j-1} q(k+1-q)+\sum_{m=j}^{k-1}(m+1)(k+1-m)=\sum_{q=1}^{k-1} q(k+1-q)+\sum_{m=j}^{k-1}(k+1-m)
\end{aligned}
$$

which coincides with the desired result (2.4) for $\alpha_{j}$, in the case $1 \leq j \leq k-1$. For the extreme cases $j=0$ and $j=k$, we obtain

$$
\begin{aligned}
& \alpha_{0}=\sum_{m=0}^{k-1}(m+1)(k+1-m)=\sum_{q=1}^{k-1} q(k+1-q)+\sum_{m=0}^{k-1}(k+1-m) \\
& \alpha_{k}=\sum_{q=1}^{k-1} q(k+1-q)
\end{aligned}
$$

which both can be incorporated in the formula (2.4) for $\alpha_{j}$. Finally, Cramer's rule gives the expression (2.3) for $c_{j}$.

Now, taking into account that $\psi \geq 0$, by assumption, and the fact that $|1+\epsilon P(\epsilon)|>|1-| \epsilon P(\epsilon)||=$ $1-\epsilon|P(\epsilon)|>0$ for all sufficiently small epsilon, we obtain 


$$
\int_{\mathbb{R}^{d}}|\varphi(x)| d x \leq \sum_{\jmath=0}^{k}\left|c_{j}\right| \epsilon^{\jmath} \leq \sum_{\jmath=0}^{k} \frac{\epsilon^{\jmath+\alpha}\left(1+\epsilon\left|P_{\jmath}(\epsilon)\right|\right)}{\epsilon^{\beta}(1-\epsilon|P(\epsilon)|)}
$$

and this latter expression can be made smaller than $1+\delta$ for sufficiently small $\epsilon$ if a) $j+\alpha, \beta>0$ for $0 \leq j \leq k-1$, and b) $k+\alpha_{k}-\beta=0$. Now, b) is obvious, as for a), we have:

$$
j+\alpha_{j}-\beta=j+\sum_{m=j}^{k-1}(k+1-m)-k=\frac{1}{2}(k-j)(k-j+1)>0,
$$

for $0 \leq j \leq k-1$. To generalize the result for arbitrary dimension $d$, it suffices to consider a product of functions of one real variable. The proof is complete.

\section{NONSTANDARD DELTA FUNCTIONS}

We prove the existence of a nonstandard function $D$ in ${ }^{*} \mathcal{D}\left(\mathbb{R}^{d}\right)$ with special properties. The proof is based on the result of Lemma 2.2 and the Saturation Principle (T. Todorov [6], p. 687). We also consider a type of nonstandard cut-off-functions which have close counterparts in standard analysis. The applications of these functions are left for the next sections.

LEMMA 3.1 (Nonstandard Mollifiers). For any positive infinitesimal $\rho$ in * $R$ there exists a nonstandard function $\theta$ in ${ }^{*} \mathcal{D}\left(\mathbb{R}^{d}\right)$ with values in ${ }^{*} \mathbb{R}$, which is symmetric and which satisfies the following properties:

(i) $\theta(x)=0$ for $\quad x \in{ }^{*} \mathbf{R}^{d}, \quad\|x\| \geq 1$;

(ii) $\int_{\mathbf{R}^{d}} \theta(x) d x=1$;

(iii) $\int_{\mathbb{R}^{d}} \theta(x) x^{\alpha} d x=0$ for all $\alpha \in \mathbb{N}_{0}^{d}, \alpha \neq 0$;

(iv) $\int_{\mathbb{R}^{d}}|\theta(x)| d x \approx 1$;

(v) $|\ln \rho|^{-1}\left(\sup _{x \in \mathbb{R}^{d}}\left|\partial^{\alpha} \theta(x)\right|\right) \approx 0$ for all $\alpha \in \mathbb{N}_{0}^{d}$;

where $\approx$ is the infinitesimal relation in ${ }^{*} \mathbb{C}$. We shall call this type of function nonstandard $\rho$-mollifiers.

PROOF. For any $k \in \mathbb{N}$, we define the set of test functions:

$$
\begin{aligned}
\bar{A}_{k}=\{ & \left\{\in \mathcal{D}\left(\mathbf{R}^{d}\right): \varphi\right. \text { is real-valued and symmetric, } \\
& \varphi(x)=0 \text { for }\|x\| \geq 1, \int_{\mathbf{R}^{d}} \varphi(x) d x=1, \\
& \left.\int_{\mathbf{R}^{d}} x^{\alpha} \varphi(x) d x=0 \text { for } 1 \leq|\alpha| \leq k, \int_{\mathbb{R}^{d}}|\varphi(x)| d x<1+\frac{1}{k}\right\}
\end{aligned}
$$

and the internal subsets of ${ }^{*} \mathcal{D}\left(\mathbf{R}^{d}\right)$ :

$$
\mathcal{A}_{k}=\left\{\varphi \in \in^{*}\left(\bar{A}_{k}\right):|\ln \rho|^{-1}\left(\sup _{x \in \mathbb{R}^{d}}\left|\partial^{\alpha}\left({ }^{*} \varphi(x)\right)\right|\right)<\frac{1}{k} \text { for }|\alpha| \leq k\right\} .
$$

Obviously, we have $\bar{A}_{1} \supset \bar{A}_{2} \supset \bar{A}_{3} \supset \ldots$ and $\mathcal{A}_{1} \supset \mathcal{A}_{2} \supset \mathcal{A}_{3} \supset \ldots$. . Also we have $\bar{A}_{k} \neq \emptyset$ for all $k \in \mathbb{N}$, by Lemma 2.2. On the other hand, we have $\bar{A}_{k} \subset \mathcal{A}_{k}$ in the sense that $\varphi \in \bar{A}_{k}$ implies * $\varphi \in \mathcal{A}_{k}$, since

$$
\sup _{x \in \mathbb{R}^{d}}\left|\partial^{\alpha}\left({ }^{*} \varphi(x)\right)\right|=\sup _{x \in \mathbb{R}^{d}}\left|\partial^{\alpha} \varphi(x)\right|=\sup _{x \leq 1}\left|\partial^{\alpha} \varphi(x)\right|
$$

is a real (standard) number and, hence, $|\ln \rho|^{-1}\left(\sup _{x \in \cdot \mathbf{R}^{d}}\left|\partial^{\alpha}\left({ }^{*} \varphi(x)\right)\right|\right)$ is infinitesimal. Thus, we have $\mathcal{A}_{k} \neq \emptyset$ for all $k$ in $\mathbb{N}$. By the Saturation Principle (T. Todorov [6], p. 687), the intersection $\mathcal{A}=\bigcap_{k \in \mathbb{N}} \mathcal{A}_{k}$ is non-empty and thus, any $\theta$ in $\mathcal{A}$ has the desired properties. 
DEFINITION 3.2 ( $\rho$-Delta Function). Let $\rho$ be a positive infinitesimal. A nonstandard function $D$ in ${ }^{*} \mathcal{D}\left(\mathbb{R}^{d}\right)$ is called a $\rho$-delta function if it takes values in " $\mathbb{R}$, it is symmetric and it satisfies the following properties:

(i) $D(x)=0$ for $x \in{ }^{*} \mathbb{R}^{d},\|x\| \geq \rho$,

(ii) $\int_{\mathbb{R}^{d}} D(x) d x=1$,

(iii) $\int_{\mathbb{R}^{d}} D(x) x^{\alpha} d x=0$ for all $\alpha \in \mathbb{N}_{0}^{d}, \alpha \neq 0$,

(iv) $\int_{\mathbb{R}^{d}}|D(x)| d x \approx 1$,

(v) $|\ln \rho|^{-1}\left(\rho^{d+|\alpha|} \sup _{x \in \mathbb{R}^{d}}\left|\partial^{\alpha} D(x)\right|\right) \approx 0$ for all $\alpha \in \mathbb{N}_{0}^{d}$.

THEOREM 3.3 (Existence). For any positive infinitesimal $\rho$ in ${ }^{*} \mathbb{R}$ there exists a $\rho$-delta function.

PROOF. Let $\theta$ be a nonstandard $\rho$-mollifier of the type described in Lemma 3.1. Then the nonstandard function $D$ in ${ }^{*} \mathcal{D}\left(\mathbb{R}^{d}\right)$, defined by

$$
D(x)=\rho^{-d} \theta(x / \rho), \quad x \in \mathbb{R}^{d},
$$

satisfies (i)-(v).

REMARK. The existence of nonstandard functions $D$ in ${ }^{*} \mathcal{D}\left(\mathbf{R}^{d}\right)$ with the above properties is in sharp contrast with the situation in standard analysis where there is no $D$ in $\mathcal{D}\left(\mathbb{R}^{d}\right)$ which satisfies both (ii) and (iii). Indeed, if we assume that $D$ is in $\mathcal{D}\left(\mathbf{R}^{d}\right)$, then (iii) implies $\widehat{D}^{(n)}(0)=0$, for all $n=1,2, \ldots$, where $\widehat{D}$ denotes the Fourier transform of $D$. It follows $\widehat{D}=\widehat{D}(0)=c$ for some constant $c$ since $\widehat{D}$ is an entire function on $\mathbb{C}^{d}$, by the Paley-Wiener Theorem (H. Bremermann [12], Theorem 8.28, p. 97). On the other hand, $D \in \mathcal{D}\left(\mathbb{R}^{d}\right) \subset \mathcal{S}\left(\mathbb{R}^{d}\right)$ implies $\hat{D} \mid \mathbb{R}^{d} \in \mathcal{S}\left(\mathbf{R}^{d}\right)$ since $\mathcal{S}\left(\mathbf{R}^{d}\right)$ is closed under Fourier transform. Thus, it follows $c=0$, i.e. $\widehat{D}=0$ which implies $D=0$ contradicting (ii).

For other classes of nonstandard delta functions we refer to (A. Robinson [15], p. 133) and to (T. Todorov [16]).

Our next task is to show the existence of an internal cut-off function.

NOTATIONS. Let $\Omega$ be an open set of $\mathbb{R}^{d}$.

1) For any $\epsilon \in \mathbb{R}_{+}$we define

$$
B_{\epsilon}=\left\{x \in \mathbb{R}^{d}:\|x\| \leq \epsilon\right\} \text { and } \Omega_{\epsilon}=\{x \in \Omega: d(x, \partial \Omega) \geq \epsilon\},
$$

where $\|x\|$ is the Euclidean norm in $\mathbb{R}^{d}, \partial \Omega$ is the boundary of $\Omega$ and $d(x, \partial \Omega)$ is the Euclidean distance between $x$ and $\partial \Omega$. We also denote:

$$
\mathcal{D}_{\epsilon}(\Omega)=\left\{\varphi \in \mathcal{D}(\Omega): \operatorname{supp} \varphi \subseteq B_{\epsilon}\right\}, E_{\epsilon}^{\prime}(\Omega)=\left\{T \in E^{\prime}(\Omega): \operatorname{supp} T \subseteq \Omega_{\epsilon}\right\} .
$$

2) We shall use the same notation, $*$, for the convolution operator $*: \mathcal{D}^{\prime}\left(\mathbb{R}^{d}\right) \times \mathcal{D}\left(\mathbb{R}^{d}\right) \rightarrow E\left(\mathbb{R}^{d}\right)$ (V. Vladimirov [13]) and its nonstandard extension *: ${ }^{*} \mathcal{D}^{\prime}\left(\mathbb{R}^{d}\right) \times{ }^{*} \mathcal{D}\left(\mathbb{R}^{d}\right) \rightarrow{ }^{*} E\left(\mathbb{R}^{d}\right)$ as well as for the convolution operator $*: E_{\epsilon}^{\prime}(\Omega) \times \mathcal{D}_{\epsilon}(\Omega) \rightarrow \mathcal{D}(\Omega)$, defined for all sufficiently small $\epsilon \in \mathbb{R}_{+}$, and for its nonstandard extension: $*:{ }^{*} E_{\epsilon}^{\prime}(\Omega) \times{ }^{*} \mathcal{D}_{\epsilon}(\Omega) \rightarrow{ }^{*} \mathcal{D}(\Omega), \epsilon \in{ }^{*} \mathbb{R}_{+}, \epsilon \approx 0$.

3) Let $\tau$ be the usual Euclidean topology on $\mathbb{R}^{d}$. We denote by $\tilde{\Omega}$ the set of the nearstandard points in ${ }^{*} \Omega$, i.e.

$$
\widetilde{\Omega}=\bigcup_{x \in \Omega} \mu(x)
$$

where $\mu(x), x \in \mathbf{R}^{d}$, is the system of monads of the topological space $\left(\mathbb{R}^{d}, \tau\right)$ (T. Todorov [6], p. 687). Recall that if $\xi \in{ }^{*} \Omega$, then $\xi \in \widetilde{\Omega}$ if and only if $\xi$ is a finite point whose standard part belongs to $\Omega$.

LEMMA 3.4. For any positive infinitesimal $\rho$ in ${ }^{*} R$ there exists a function $\Pi$ in ${ }^{*} \mathcal{D}(\Omega)$ (a $\rho$-cut-off function) such that:

a) $\Pi(x)=1$ for all $x \in \widetilde{\Omega}$;

b) $\operatorname{supp} \Pi \subseteq{ }^{*} \Omega_{\rho}$, where ${ }^{*} \Omega_{\rho}=\left\{\xi \in{ }^{*} \Omega:{ }^{*} d(\xi, \partial \Omega) \geq \rho\right\}$. 
PROOF. Let $\rho$ be a positive infinitesimal in ${ }^{*} \mathbf{R}$ and $D$ be a $\rho$-delta function Define the internal set $X=\left\{\xi \in{ }^{*} \Omega:{ }^{*}\|\xi\| \leq 1 / \rho,{ }^{*} d(\xi, \partial \Omega) \geq 2 \rho\right\}$ and let $\chi$ be its characteristic function. Then the function $\Pi=\chi * D$ has the desired property.

\section{THE ALGEBRA OF ASYMPTOTIC FUNCTIONS}

We define and study the algebra ${ }^{P} E(\Omega)$ of asymptotic functions on an open set $\Omega$ of $\mathbb{R}^{d}$. The construction of the algebra ${ }^{\rho} E(\Omega)$, presented here, is a generalization and a refinement of the constructions in [9] and [10] (by the authors of this paper, respectively), where the algebra ${ }^{\rho} E\left(\mathbb{R}^{d}\right)$ was introduced by somewhat different but equivalent definitions. On the other hand, the algebra of asymptotic functions ${ }^{P} E(\Omega)$ is somewhat similar to but different from the J. F. Colombeau [7], [8] algebras of new generalized functions. This essential difference between $P E(\Omega)$ and J. F. Colombeau's algebras of generalized functions is the properties of the generalized scalars: the scalars of the algebra ${ }^{\rho} E(\Omega)$ constitutes an algebraically closed field (as any scalars should do) while the scalars of J. F. Colombeau's algebras are rings with zero divisors (J. F. Colombeau [8], §2.1). This improvement compared with J. F. Colombeau's theory is due to the involvement of the nonstandard analysis.

Let $\Omega$ be an open set of $\mathbb{R}^{d}$ and $\rho \in{ }^{*} \mathbf{R}$ be a positive infinitesimal. We shall keep $\Omega$ and $\rho$ fixed in what follows.

Following A. Robinson [2], we define:

DEFINITION 4.1 (Robinson's Asymptotic Numbers). The field of the complex Robinson $\rho$ asymptotic numbers is defined as the factor space ${ }^{\rho} \mathbb{C}=\mathbb{C}_{M} / \mathbb{C}_{0}$, where

$$
\begin{aligned}
\mathbb{C}_{M} & =\left\{\xi \in \in^{*} \mathbb{C}:|\xi|<\rho^{-n} \text { for some } n \in \mathbb{N}\right\}, \\
\mathbb{C}_{0} & =\left\{\xi \in \in^{*} \mathbb{C}:|\xi|<\rho^{n} \text { for all } n \in \mathbb{N}\right\},
\end{aligned}
$$

(" $M$ " stands for "moderate"). We define the embedding $\mathbb{C} \subset{ }^{\rho} \mathbb{C}$ by $c \rightarrow q(c)$, where $q: \mathbb{C}_{M} \rightarrow{ }^{\rho} \mathbb{C}$ is the quotient mapping. The field of the real asymptotic numbers is defined by ${ }^{\rho} \mathbb{R}=q\left({ }^{*} \mathbb{R} \cap \mathbb{C}_{M}\right)$.

It is easy to check that $\mathbb{C}_{0}$ is a maximal ideal in $\mathbb{C}_{M}$ and hence ${ }^{\rho} \mathbb{C}$ is a field. Also ${ }^{\rho} \mathbb{R}$ is a real closed totally ordered nonarchimedean field (since ${ }^{*} \mathbb{R}$ is a real closed totally ordered field) containing $\mathbb{R}$ as a totally ordered subfield. Thus, it follows that ${ }^{P} \mathbb{C}={ }^{\rho} \mathbf{R}(i)$ is an algebraically closed field, where $i=\sqrt{-1}$.

The algebra of "asymptotic functions" is, in a sense, a $C^{\infty}$-counterpart of A. Robinson's asymptotic numbers ${ }^{\rho} \mathbb{C}$ :

DEFINITION 4.2 (Asymptotic Functions on $\Omega$ ). (i) We define the class ${ }^{\rho} E(\Omega)$ of the $\rho$-asymptotic functions on $\Omega$ (or simply, asymptotic functions on $\Omega$ if no confusion 'could arise) as the factor space ${ }^{\rho} E(\Omega)=E_{M}(\Omega) / E_{0}(\Omega)$, where

$$
\begin{aligned}
E_{M}(\Omega) & =\left\{f \in{ }^{*} E(\Omega): \partial^{\alpha} f(\xi) \in \mathbb{C}_{M}, \text { for all } \alpha \in \mathbb{N}_{0}^{d} \text { and all } \xi \in \widetilde{\Omega}\right\}, \\
E_{0}(\Omega) & \left.=\left\{f \in{ }^{*} E(\Omega): \partial^{\alpha} f(\xi)\right\} \in \mathbb{C}_{0}, \text { for all } \alpha \in \mathbb{N}_{0}^{d} \text { and all } \xi \in \tilde{\Omega}\right\},
\end{aligned}
$$

and $\widetilde{\Omega}$ is the set of the nearstandard points of ${ }^{*} \Omega$ (3.2). The functions in $E_{M}(\Omega)$ are called $\rho$-moderate (or, simply, moderate) and those in $E_{0}(\Omega)$ are called $\rho$-mull functions (or, simply, mull functions).

(ii) The pairing between ${ }^{\rho} E(\Omega)$ and $\mathcal{D}(\Omega)$ with values in ${ }^{\rho} \mathbb{C}$, is defined by

$$
\left\langle Q_{\Omega}(f), \varphi\right\rangle=q\left(\int_{\cdot_{\Omega}} f(x){ }^{*} \varphi(x) d x\right),
$$

where $q: \mathbb{C}_{M} \rightarrow{ }^{\rho} \mathbb{C}$ and $Q_{\Omega}: E_{M}(\Omega) \rightarrow{ }^{\rho} E(\Omega)$ are the corresponding quotient mappings, $\varphi$ is in $\mathcal{D}(\Omega)$ and ${ }^{*} \varphi$ is its nonstandard extension.

(iii) We define the canonical embedding $E(\Omega) \subset{ }^{\rho} E(\Omega)$ by the mapping $\sigma_{\Omega}: f \rightarrow Q_{\Omega}\left({ }^{*} f\right)$, where * $f$ is the nonstandard extension of $f$. 
EXAMPLE 4.3. Let $D$ be a nonstandard $\rho$-delta function in the sense of Definition 3.2. Then $D \in E_{M}\left(\mathbb{R}^{d}\right)$. In addition, $\left.D\right|^{*} \Omega \in E_{M}(\Omega)$, where $\left.D\right|^{*} \Omega$ denotes the pointwise restriction of $D$ on ${ }^{*} \Omega$. To show this, denote $|\ln \rho|^{-1}\left(\rho^{d+|\alpha|} \sup _{x \in \mathbb{R}^{d}}\left|\partial^{\alpha} D(x)\right|\right)=h_{\alpha}$ and observe that $h_{\alpha} \approx 0$ for all $\alpha \in \mathbb{N}_{0}^{d}$, by the definition of $D$. Thus, for any (finite) $x$ in ${ }^{*} \mathbb{R}^{d}$ and any $\alpha \in \mathbb{N}_{0}^{d}$ we have $\left|\partial^{\alpha} D(x)\right| \leq$ $\sup _{x \in \mathbb{R}^{d}}\left|\partial^{\alpha} D(x)\right|=\frac{h_{a}|\ln \rho|}{\rho^{d+|\alpha|}}<\rho^{-n}$, for $n=d+|\alpha|+1$, thus, $D \in E_{M}\left(\mathbb{R}^{d}\right)$. On the other hand, $\left.D\right|^{*} \Omega \in E_{M}(\Omega)$ follows immediately from the fact that $\widetilde{\Omega}$ consists of finite points in $" \mathbb{R}^{d}$ only.

THEOREM 4.4 (Differential Algebra). (i) The class of asymptotic functions ${ }^{\rho} E(\Omega)$ is a differential algebra over the field of the complex asymptotic numbers ${ }^{\rho} \mathbb{C}$.

(ii) $E(\Omega)$ is a differential subalgebra of ${ }^{\rho} E(\Omega)$ over the scalars $\mathbb{C}$ under the canonical embedding $\sigma_{\Omega}$. In addition, $\sigma_{\Omega}$ preserves the pairing in the sense that $\langle f, \varphi\rangle=\left\langle\sigma_{\Omega}(f), \varphi\right\rangle$ for all $f$ in $E(\Omega)$ and for all $\varphi$ in $\mathcal{D}(\Omega)$, where $\langle f, \varphi\rangle=\int_{\Omega} f(x) \varphi(x) d x$ is the usual pairing between $E(\Omega)$ and $\mathcal{D}(\Omega)$.

PROOF. (i) It is clear that $E_{M}(\Omega)$ is a differential ring and $E_{0}(\Omega)$ is a differential ideal in $E_{M}(\Omega)$ since $\mathbb{C}_{M}$ is a ring and $\mathbb{C}_{0}$ is an ideal in $\mathbb{C}_{M}$ and, on the other hand, both $E_{M}(\Omega)$ and $E_{0}(\Omega)$ are closed under differential, by definition. Hence, the factor space ${ }^{\rho} E(\Omega)$ is also a differential ring. It is clear that, $E_{M}(\Omega)$ is a module over the ring $\mathbb{C}_{M}$ and, in addition, the annihilator $\left\{c \in \mathbb{C}_{M}: c f \in E_{0}(\Omega), f \in E_{M}(\Omega)\right\}$ of $\mathbb{C}_{M}$ coincides with the ideal $\mathbb{C}_{0}$. Thus, ${ }^{\rho} E(\Omega)$ becomes an algebra over the field of the complex asymptotic numbers ${ }^{\rho} \mathbb{C}$.

(ii) Assume that $\sigma_{\Omega}\left({ }^{*} f\right)=0$ in ${ }^{\rho} E(\Omega)$, i.e. ${ }^{*} f \in E_{0}(\Omega)$. By the definition of $E_{0}(\Omega)$ (applied for $\alpha=0$ and $n=1$ ), it follows $f=0$ since ${ }^{*} f$ is an extension of $f$ and $\rho$ is an infinitesimal. Thus, the mapping $f \rightarrow \sigma_{\Omega}(f)$ is injective. It preserves the algebraic operations since the mapping $f \rightarrow{ }^{*} f$ preserves them. The preserving of the pairing follows immediately from the fact that $\int_{{ }_{\Omega}}{ }^{*} f(x) d x=$ $\int_{\Omega} f(x) d x$, by the Transfer Principle (T. Todorov [6], p. 686). The proof is complete.

\section{EMBEDDING OF SCHWARTZ DISTRIBUTIONS}

Let $\Omega$ be (as before) an open set of $\mathbf{R}^{d}$. Recall that the Schwartz embedding $L_{\Omega}: \mathcal{L}_{\text {loc }}(\Omega) \rightarrow \mathcal{D}^{\prime}(\Omega)$ from $\mathcal{L}_{\text {loc }}(\Omega)$ into $\mathcal{D}^{\prime}(\Omega)$ is defined by the formula:

$$
\left\langle L_{\Omega}(f), \varphi\right\rangle=\int_{\Omega} f(x) \varphi(x) d x, \quad \varphi \in \mathcal{D}(\Omega) .
$$

Here $\mathcal{L}_{\text {loc }}(\Omega)$ denotes, as usual, the space of the locally (Lebesgue) integrable complex valued functions on $\Omega$ (V. Vladimirov [13]). The Schwartz embedding $L_{\Omega}$ preserves the addition and multiplication by a complex number, hence, the space $\mathcal{L}_{\text {loc }}(\Omega)$ can be considered as a linear subspace of $\mathcal{D}^{\prime}(\Omega)$. In addition, the restriction $L_{\Omega} \mid E(\Omega)$ of $L_{\Omega}$ on $E(\Omega)$ (often denoted also by $L_{\Omega}$ ) preserves the partial differentiation of any order and in this sense $E(\Omega)$ is a differential linear subspace of $\mathcal{D}^{\prime}(\Omega)$. In short, we have the chain of linear embeddings: $\mathcal{L}_{\text {loc }}(\Omega) \subset E(\Omega) \subset \mathcal{D}^{\prime}(\Omega)$.

The purpose of this section is to show that the algebra of asymptotic functions ${ }^{\rho} E(\Omega)$ contains an isomorphic copy of the space of Schwartz distributions $\mathcal{D}^{\prime}(\Omega)$ and, hence, to offer a solution of the Problem of Multiplication of Schwartz Distributions. This result is a generalization of some results in [9] and [10] (by the authors of this paper, respectively) where only the embedding of the tempered distributions $\mathcal{S}^{\prime}\left(\mathbb{R}^{d}\right)$ in ${ }^{\rho} E\left(\mathbb{R}^{d}\right)$ has been established. The embedding of all distributions $\mathcal{D}^{\prime}(\Omega)$, discussed here, presents an essentially different situation.

The spaces $\widetilde{E}(\Omega)$ and $\widetilde{\mathcal{D}}(\Omega)$, defined below, are immediate generalizations of the spaces $\widetilde{E}\left(\mathbb{R}^{d}\right)$ and $\widetilde{\mathcal{D}}\left(\mathbf{R}^{d}\right)$, introduced in (K. D. Stroyan and W. A. Luxemburg [17], (10.4), p. 299):

$$
\begin{aligned}
\widetilde{E}(\Omega)=\left\{\varphi \in{ }^{*} E(\Omega):\right. & \partial^{\alpha} \varphi(x) \text { is a finite number in }{ }^{*} \mathbb{C} \text { for all } \\
& \left.x \in \widetilde{\Omega} \text { and all } \alpha \in \mathbb{N}_{0}^{d}\right\},
\end{aligned}
$$




$$
\begin{aligned}
\widetilde{\mathcal{D}}(\Omega)=\left\{\varphi \in{ }^{*} E(\Omega):\right. & \partial^{\alpha} \varphi(x) \text { is a finite number in " } \mathbb{C} \text { for all } \\
& \left.x \in \widetilde{\Omega}, \alpha \in \mathbb{N}_{0}^{d} \text { and } \varphi(x)=0 \text { for all } x \in{ }^{*} \Omega \backslash \widetilde{\Omega}\right\},
\end{aligned}
$$

Obviously, we have $\widetilde{\mathcal{D}}(\Omega) \subset \widetilde{E}(\Omega) \subset E_{M}(\Omega)$. Notice as well that $\varphi \in \widetilde{\mathcal{D}}(\Omega)$ implies $\varphi \in{ }^{*} \mathcal{D}(G)$ for some open relatively compact set $G$ of $\Omega$. We have also the following simple result:

LEMMA 5.1. If $T \in \mathcal{D}^{\prime}(\Omega)$ and $\varphi \in E_{0}(\Omega) \cap \widetilde{\mathcal{D}}(\Omega)$, then $\left\langle{ }^{*} T, \varphi\right\rangle \in \mathbb{C}_{0}$.

PROOF. Observe that $E_{0}(\Omega) \cap \widetilde{\mathcal{D}}(\Omega)$ implies $\varphi \in E_{0}(\Omega) \cap{ }^{*} \mathcal{D}(G)$ for some open relatively compact set $G$ of $\Omega$. By the continuity of $T$ (and Transfer Principle) there exist constants $M \in \mathbb{R}_{+}$and $m \in \mathbb{N}_{0}$ such that

$$
\left|\left\langle^{*} T, \varphi\right\rangle\right| \leq M \sum_{|\mu| \leq m} \sup _{x \in \in^{*} G}\left|\partial^{\mu} \varphi(x)\right|
$$

On the other hand, $M \sum_{|\mu| \leq m} \sup _{x \in \cdot G}\left|\partial^{\mu} \varphi(x)\right|<\rho^{n}$ for all $n \in \mathbb{N}$, since $\varphi \in E_{0}(\Omega)$, by assumption. Thus, $\left|\left\langle^{*} T, \varphi\right\rangle\right|<\rho^{n}$ for all $n \in \mathbb{N}$.

Let $D$ be a $\rho$-delta function in the sense of Definition 3.2. We shall keep $D$ (along with $\Omega$ and $\rho$ ) fixed in what follows.

DEFINITION 5.2 (Embedding of Schwartz Distributions). We define the embedding $\mathcal{D}^{\prime}(\Omega) \subset{ }^{\rho} E(\Omega)$ by $\Sigma_{D, \Omega}: T \rightarrow Q_{\Omega}\left(\left({ }^{*} T \Pi_{\Omega}\right) * D\right)$, where ${ }^{*} T$ is the nonstandard extension of $T, \Pi_{\Omega}$ is a (an arbitrarily chosen) $\rho$-cut-off function for $\Omega$ (Lemma 3.4), ${ }^{*} T \Pi_{\Omega}$ is the Schwartz product between ${ }^{*} T$ and $\Pi_{\Omega}$ in ${ }^{*} \mathcal{D}^{\prime}(\Omega)$ (defined by Transfer Principle), $*$ is the convolution operator and $Q_{\Omega}: E_{M}(\Omega) \rightarrow{ }^{\rho} E(\Omega)$ is the quotient mapping in the definition of ${ }^{\rho} E(\Omega)$ (Definition 4.2).

The cut-off function $\Pi_{\Omega}$ can be dropped in the above definition, i.e. $\Sigma_{D, \Omega}: T \rightarrow Q_{\Omega}\left({ }^{*} T * D\right)$, in some particular cases; e.g. when:

a) $T$ has a compact support in $\Omega$;

b) $\Omega=\mathbb{R}^{d}$.

PROPOSITION 5.3 (Correctness). $T \in \mathcal{D}^{\prime}(\Omega)$ implies $\left({ }^{*} T \Pi_{\Omega}\right) * D \in E_{M}(\Omega)$.

PROOF. Choose $\alpha \in \mathbb{N}_{0}^{d}$ and all $x \in \tilde{\Omega}$. Since we have $\partial^{\alpha}\left(\left(\Pi_{\Omega}{ }^{*} T\right) * D\right)(x)=\left(\partial^{\alpha}\left({ }^{*} T\right) * D\right)(x)$ (by the definition of $\Pi_{\Omega}$ ), we need to show that $\partial^{\alpha}\left({ }^{*} T * D\right)(x) \in \mathbb{C}_{M}$ only, i.e. that $\left|\partial^{\alpha}\left({ }^{*} T * D\right)(x)\right|<\rho^{-m}$ for some $m \in \mathbb{N}$ ( $m$ might depend on $\alpha$ ). We start with the case $\alpha=0$ Denote $D_{x}(\xi)=D(\xi-x), \xi \in{ }^{*} \mathbb{R}$ and observe that $\operatorname{supp}\left(D_{x}\right) \subseteq{ }^{*} G$ for some open relatively compact set $G$ of $\Omega$, since $D_{x}$ vanishes on ${ }^{*} \Omega \backslash \widetilde{\Omega}$. Next, by the continuity of $T$ (and the Transfer Principle), there exist constants $m \in \mathbb{N}_{0}$ and $M \in \mathbf{R}_{+}$such that

$$
\left|\left({ }^{*} T * D\right)(x)\right|=\left|\left\langle^{*} T,\left.D_{x}\right|^{*} \Omega\right\rangle\right| \leq M \sum_{|\mu| \leq m} \sup _{\xi \epsilon^{*} G}\left|\partial_{\xi}^{\mu} D(x-\xi)\right| .
$$

Finally, there exists $n \in \mathbb{N}$ such that $\sum_{|\mu| \leq m} \sup _{\xi \in^{\circ} G}\left|\partial_{\xi}^{\mu} D(x-\xi)\right|<\rho^{-n}$, since $\left.D\right|^{*} G$ is a $\rho$-moderate function (Example 4.3). Combining these arguments, we have: $\left|\left({ }^{*} T * D\right)(x)\right| \leq M \rho^{-n}<\rho^{-(n+1)}$, as required. The generalization for arbitrary multiindex $\alpha$ follows immediately since $\partial^{\alpha}\left({ }^{*} T * D\right)=$ $\left(\partial^{\alpha}\left({ }^{*} T\right)\right) * D={ }^{*}\left(\partial^{\alpha} T\right) * D$, by Transfer Principle, a $\partial^{\alpha} T$ is (also) in $\mathcal{D}^{\prime}(\Omega)$.

PROPOSITION 5.4. $f \in \widetilde{\mathrm{E}}(\Omega)$ implies $\left(f \Pi_{\Omega}\right) * D-f \in \mathrm{E}_{0}(\Omega)$.

PROOF. Let $x \in \widetilde{\Omega}$ and $\alpha \in \mathbb{N}_{0}^{d}$. Since we have $\partial^{\alpha}\left[\left(\left(f \Pi_{\Omega}\right) * D\right)(x)-f(x)\right]=$ $\partial^{\alpha}[(f * D)(x)-f(x)]$ (by the definition of $\Pi_{\Omega}$ ), we need to show that $\partial^{\alpha}[(f * D)(x)-f(x)] \in \mathbb{C}_{0}$ only. Choose $n \in \mathbb{N}$. We need to show that $\left|\partial^{\alpha}[(f * D)(x)-f(x)]\right|<\rho^{n}$. We start first with the case $\alpha=0$. By Taylor's formula (applied by transfer), we have 


$$
f(x-\xi)-f(x)=\sum_{|\beta|=1}^{n} \frac{(-1)^{|\beta|} \partial^{\beta} f(x)}{\beta !} \xi^{\beta}+\frac{(-1)^{n+1}}{(n+1) !} \sum_{|\beta|=n+1} \partial^{\beta} f(\eta(\xi)) \xi^{\beta}
$$

for any $\xi \in \widetilde{\Omega}$, where $\eta(\xi)$ is a point in * $\Omega$ "between $x$ and $\xi$." Notice that the point $\eta(\xi)$ is also in $\widetilde{\Omega}$ It follows

$(f * D)(x)-f(x)=\int_{\|\xi\| \leq \rho} D(\xi)[f(x-\xi)-f(x)] d \xi=\frac{(-1)^{n+1}}{(n+1) !} \sum_{|\beta|=n+1} \int_{\|\xi\| \leq \rho} D(\xi) \xi^{\beta} \partial^{\beta} f(\eta(\xi)) d \xi$,

since $\int_{\|\xi\| \leq \rho} D(\xi) \xi^{\beta} d \xi=0$, by the definition of $D$. Thus, we have

$$
|(f * D)(x)-f(x)| \leq \frac{\rho^{n+1}}{(n+1) !}\left(\int_{\mathbb{R}^{d}}|D(x)| d x\right)\left(\sum_{|\beta|=n+1} \sup _{\|\xi\| \leq \rho}\left|\partial^{\beta} f(\eta(\xi))\right|\right)<\rho^{n},
$$

as desired, since, on one hand, $\int_{*_{\mathbb{R}^{d}}}|D(x)| d x \approx 1$, by the definition of $D$ and on the other hand, the above sum is a finite number because * $\partial^{\beta} f(\eta(\xi))$ are all finite due to $\eta(\xi) \in \widetilde{\Omega}$. The generalization for an arbitrary $\alpha$ is immediate since $\partial^{\alpha}[(f * D)(x)-f(x)]=\left(\partial^{\alpha} f * D\right)(x)-\partial^{\alpha} f(x)$, by the Transfer Principle.

COROLLARY 5.5. (i) $f \in E(\Omega)$ implies $\left(* f \Pi_{\Omega}\right) * D-{ }^{*} f \in E_{0}(\Omega)$.

(ii) $\varphi \in \mathcal{D}(\Omega)$ implies $\left({ }^{*} \varphi \Pi_{\Omega}\right) * D-{ }^{*} \varphi \in E_{0}(\Omega) \cap \widetilde{\mathcal{D}}(\Omega)$.

PROOF. (i) follows immediately from the above proposition since $f \in E(\Omega)$ implies * $f \in \widetilde{E}(\Omega)$.

(ii) Both $\left({ }^{*} \varphi \Pi_{\Omega}\right) * D$ and ${ }^{*} \varphi$ vanish on ${ }^{*} \Omega \backslash \widetilde{\Omega}$ since their supports are within an open relatively compact neighborhood $G$ of $\operatorname{supp}(\varphi)$ and the latter is a compact set of $\Omega$, by assumption. Thus,

$$
\left({ }^{*} \varphi \Pi_{\Omega}\right) * D-{ }^{*} \varphi \in{ }^{*} \mathcal{D}(G) \subset \widetilde{\mathcal{D}}(\Omega), \quad \text { as required. }
$$

Denote $\check{D}(x)=D(-x)$ and recall that $\check{D}=D$ since $D$ is symmetric (Definition 3.2).

PROPOSITION 5.6. If $T \in \mathcal{D}^{\prime}(\Omega)$ and $\varphi \in \mathcal{D}(\Omega)$, then

$$
\int_{{ }^{\prime}}\left(\left({ }^{*} T \Pi_{\Omega}\right) * D\right)(x)^{*} \varphi(x) d x-\langle T, \varphi\rangle \in \mathbb{C}_{0}
$$

PROOF. Using the properties of the convolution operator (applied by transfer), we have

$$
\begin{aligned}
\left.\int_{{ }^{*}}\left({ }^{*} T \Pi_{\Omega}\right) * D\right)(x){ }^{*} \varphi(x) d x-\langle T, \varphi\rangle \\
\quad=\left\langle\left({ }^{*} T \Pi_{\Omega}\right) * D,{ }^{*} \varphi\right\rangle-\left\langle{ }^{*} T,{ }^{*} \varphi\right\rangle=\left\langle{ }^{*} T \Pi_{\Omega},{ }^{*} \varphi * \check{D}\right\rangle-\left\langle{ }^{*} T \Pi_{\Omega},{ }^{*} \varphi\right\rangle \\
\quad=\left\langle{ }^{*} T \Pi_{\Omega},{ }^{*} \varphi * \check{D}-{ }^{*} \varphi\right\rangle=\left\langle{ }^{*} T,{ }^{*} \varphi * D-{ }^{*} \varphi\right\rangle \in \mathbb{C}_{0},
\end{aligned}
$$

by Lemma 5.1 since ${ }^{*} \varphi * D-{ }^{*} \varphi \in E_{0}(\Omega) \cap \widetilde{\mathcal{D}}(\Omega)$, by Corollary 5.5 .

We are ready to state our main result:

THEOREM 5.7 (Properties of $\Sigma_{D, \Omega}$ ). (i) $\Sigma_{D, \Omega}$ preserves the pairing in the sense that for all $T$ in $\mathcal{D}^{\prime}(\Omega)$ and all $\varphi$ in $\mathcal{D}(\Omega)$ we have $\langle T, \varphi\rangle=\left\langle\Sigma_{D, \Omega}(T), \varphi\right)$, where the left hand side is the (usual) pairing of $T$ and $\varphi$ in $\mathcal{D}^{\prime}(\Omega)$, while the right hand side is the pairing of $\Sigma_{D, \Omega}(T)$ and $\varphi$ in ${ }^{\rho} E(\Omega)$ (Definition 4.2).

(ii) $\Sigma_{D, \Omega}$ is injective and it preservers all linear operations in $\mathcal{D}^{\prime}(\Omega)$ : the addition, multiplication by (standard) complex numbers and the partial differentiation of any (standard) order.

(iii) $\Sigma_{D, \Omega}$ is an extension of the canonical embedding $\sigma_{\Omega}$ defined earlier in Definition 4.2 in the sense that $\sigma_{\Omega}=\Sigma_{D, \Omega} \circ L_{\Omega}$, where $L_{\Omega}$ is the Schwartz embedding (5.1) restricted on $E(\Omega)$ and $\circ$ denotes composition. Or, equivalently, the following diagram is commutative: 


$$
\begin{aligned}
& \mathcal{D}^{\prime}(\Omega) \\
& L_{\Omega} \nearrow \\
& E(\Omega) \quad \downarrow \Sigma_{D, \Omega} \\
& \sigma_{\Omega} \searrow \\
& { }^{\rho} E(\Omega) .
\end{aligned}
$$

PROOF. (i) Denote (as before) $\check{D}(x)=D(-x)$ and recall that $\check{D}(x)=D$ (Definition 3.2). We have

$$
\begin{aligned}
\left\langle\Sigma_{D, \Omega}(T), \varphi\right\rangle & \left.=\left\langle Q_{\Omega}\left({ }^{*} T \Pi_{\Omega}\right) * D\right), \varphi\right\rangle-\langle T, \varphi\rangle \\
& =q\left(\int_{\Omega_{\Omega}}\left(\left(\Pi_{\Omega}{ }^{*} T\right) * D\right)(x){ }^{*} \varphi(x) d x\right)-q(\langle T, \varphi\rangle) \\
& \left.=q\left(\int_{\cdot_{\Omega}}\left({ }^{*} T \Pi_{\Omega}\right) * D\right)(x)^{*} \varphi(x) d x-\langle T, \varphi\rangle\right)=0,
\end{aligned}
$$

because $\left.\int_{\cdot_{\Omega}}\left({ }^{*} T \Pi_{\Omega}\right) * D\right)(x){ }^{*} \varphi(x) d x-\langle T, \varphi\rangle \in \mathbb{C}_{0}$, by Proposition 5.6. Here $\langle T, \varphi\rangle=q(\langle T, \varphi\rangle)$ holds because $\langle T, \varphi\rangle$ is a standard (complex) number.

(ii) The injectivity of $\Sigma_{D, \Omega}$ follows from (i): $\Sigma_{D, \Omega}(T)=0$ in ${ }^{\rho} E(\Omega)$ implies $\left\langle\Sigma_{D, \Omega}(T), \varphi\right\rangle=0$ for all $\varphi \in \mathcal{D}(\Omega)$, which is equivalent to $\langle T, \varphi\rangle=0$ for all $\varphi \in \mathcal{D}(\Omega)$, by (i), thus, $T=0$ in $\mathcal{D}^{\prime}(\Omega)$, as required. The preserving of the linear operations follows from the fact that both the extension mapping * and the convolution * (applied by Transfer Principle) are linear operators.

(iii) For any $f \in E(\Omega)$ we have $\left.\sigma(f)=Q_{\Omega}\left({ }^{*} f\right)=Q_{\Omega}\left({ }^{*} f \Pi_{\Omega}\right) * D\right)=Q_{\Omega}\left(\left(^{*} L(f) \Pi_{\Omega}\right) * D\right)=$ $\Sigma_{D, \Omega}(L(f))$, as required, since $* f-\left(* f \Pi_{\Omega}\right) * D \in E_{0}(\Omega)$, by Corollary 5.5 .

REMARK 5.8 (Multiplication of Distributions). As a consequence of the above result, the Schwartz distributions in $\mathcal{D}^{\prime}(\Omega)$ can be multiplied within the associative and commutative differential algebra ${ }^{\rho} E(\Omega)$ (something impossible in $\mathcal{D}^{\prime}(\Omega)$ itself). By the property (iii) above, the multiplication in ${ }^{P} E(\Omega)$ coincides on $E(\Omega)$ with the usual (pointwise) multiplication in $E(\Omega)$. Thus, the class ${ }^{\rho} E(\Omega)$, endowed with an embedding $\Sigma_{D, \Omega}$, presents a solution of the problem of multiplication of Schwartz distributions which, in a sense, is optimal, in view of the Schwartz impossibility results (L. Schwartz [1]) (for a discussion we refer also to J. F. Colombeau [7], \$2.4 and M. Oberguggenberg [18], §2). We should mention that the existence of an embedding of $\mathcal{D}^{\prime}\left(\mathbf{R}^{d}\right)$ into ${ }^{P} E\left(\mathbf{R}^{d}\right)$ can be proved also by sheaftheoretical arguments as indicated in (M. Oberguggenberger [18], §23).

REMARK 5.9 (Nonstandard Asymptotic Analysis). We sometimes refer to the area connected directly or indirectly with the fields ${ }^{\boldsymbol{\rho}} \mathbf{R}$ as Nonstandard Asymptotic Analysis. The fields ${ }^{\boldsymbol{}} \mathbf{R}$ were introduced by $A$. Robinson [2] and are sometimes known as "Robinson's nonarchimedean valuation fields." The terminology "Robinson's asymptotic numbers," chosen in this paper, is due to the role of ${ }^{\rho} \mathbb{R}$ for the asymptotic expansions of classical functions (A. H. Lightstone and A. Robinson [3]) and also to stress the fact that in our approach ${ }^{\rho} \mathbb{C}$ plays the role of the scalars of the algebra ${ }^{\circ} E(\Omega)$. Linear spaces over the field ${ }^{\rho} \mathbf{R}$ has been studied by W. A. J. Luxemburg [19] in order to establish a connection between nonstandard and nonarchimedean analysis. More recently ${ }^{\rho} \mathbf{R}$ has been used by V. Pestov [20] for studying Banach spaces. The field ${ }^{\rho} \mathbf{R}$ has been exploited by Li Bang-He [21] for multiplication of Schwartz distributions.

\section{REFERENCES}

[1] SCHWARTZ, L., Sur l'impossibilité de la multiplication des distributions, C.R. Acad. Sci., Paris, 239 (1954), 847-848.

[2] ROBINSON, A., Function theory on some nonarchimedean fields, Amer. Math. Monthly 80 (6), Part II: Papers in the Foundations of Mathematics (1973), 87-109. 
[3] LIGHTSTONE, A.H. and ROBINSON, A., Nonarchimedean Fields and Asymptotic Expansions, North-Holland Mathematical Library, 13, North Holland, Amsterdam-Oxford/American Elsevier, New York, 1975.

[4] OBERGUGGENBERGER, M., Contributions of nonstandard analysis to partial differential equations, in Development in Nonstandard Mathematics (Eds. N.J. Cutland, V. Neves, F. Oliveira and J. Sousa-Pinto), Longman-Harlow, 1995, 130-150.

[5] TODOROV, T., An existence result for a class of partial differential equations with smooth coefficients, in Advances in Analysis, Probability and Mathematical Physics - Contributions to Nonstandard Analysis (Eds. S. Albeverio, W.A.J. Luxemburg and M.P.H. Wolff), Kluwer Academic Publishers (Mathematics and Its Applications), Dordrecht/Boston/London, Vol. 314, 1995, 107-121.

[6] TODOROV, T., An existence of solutions for linear partial differential equations with $\mathrm{C}^{\infty}$. coefficients in an algebra of generalized functions, in Trans. Am. Math. Soc., 348, 2 (1996).

[7] COLOMBEAU, J.F., New Generalized Functions and Multiplication of Distributions, NorthHolland Math. Studies 84, North-Holland, Amsterdam, 1984.

[8] COLOMBEAU, J.F., Elementary Introduction to New Generalized Functions, North-Holland, Amsterdam, 1985.

[9] OBERGUGGENBERGER, M., Products of distributions: nonstandard methods, Z. Anal. Anwendungen 7 (1988), 347-365., Corrections: ibid. 10 (1991), 263-264.

[10] TODOROV, T., Colombeau's new generalized function and non-standard analysis, in Generalised Functions, Convergence Structures and their Applications (Eds. B. Stankovic, E. Pap, S. Pilipovic and V.S. Vladimirov), Plenum Press, New York (1988), 327-339.

[11] HOSKINS, R.F. and PINTO, J.S., Nonstandard treatments of new generalised functions, in Generalized Functions and Their Applications (Ed. R.S. Pathak), Plenum Press, New York, 1993, 95-108.

[12] BREMERMANN, H., Distributions, Complex Variables, and Fourier Transforms, AddisonWesley Publ. Co., Inc., Pal Alto, 1965.

[13] VLADIMIROV, V., Generalized Functions in Mathematical Physics, Mir-Publisher, Moscow, 1979.

[14] LINDSTROM, T., An invitation to nonstandard analysis, in Nonstandard Analysis and its Applications (Ed. N. Cutland), London Mathematical Society Student Texts 10, Cambridge University Press, Cambridge, 1988, 1-105.

[15] ROBINSON, A., Non-Standard Analysis, North-Holland, Amsterdam, 1966.

[16] TODOROV, T., A nonstandard delta function, Proc. Amer. Math. Soc., 110 (1990), 1143-1144.

[17] STROYAN, K.D. and LUXEMBURG, W.A.J., Introduction to the Theory of Infinitesimals, Academic Press, New York, 1976.

[18] OBERGUGGENBERGER, M., Multiplication of distributions and applications to partial differential equations, Pitman Research Notes Math., 259, Longman, Harlow, 1992.

[19] LUXEMBURG, W.A.J., On a class of valuation fields introduced by A. Robinson, Israel Journal Math., 25 (1976), 189-201.

[20] PESTOV, V., On a valuation field invented by A. Robinson and certain structures connected with it, Israel Journal Math., 74 (1991), 65-79.

[21] BANG-HE, L., Non-standard analysis and multiplication of distributions, Sci. Sinica, 21 (1978), 561-585. 


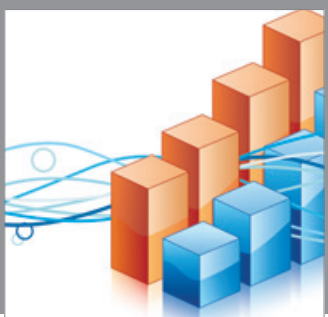

Advances in

Operations Research

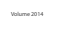

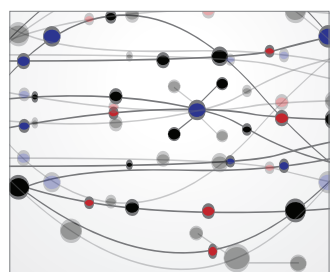

\section{The Scientific} World Journal
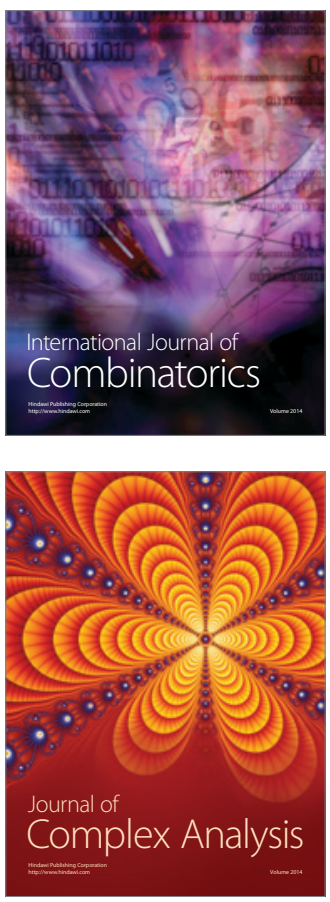

International Journal of

Mathematics and

Mathematical

Sciences
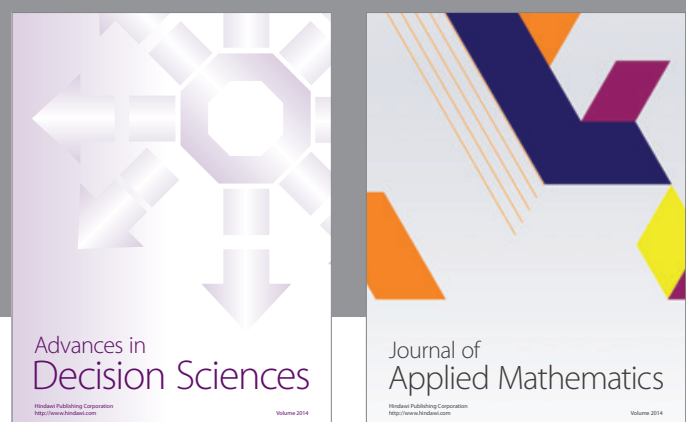

Journal of

Applied Mathematics
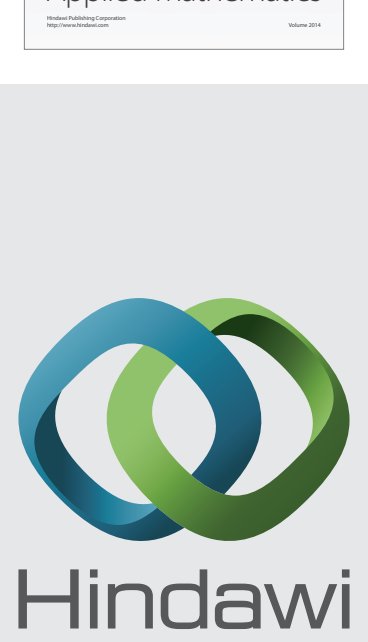

Submit your manuscripts at http://www.hindawi.com
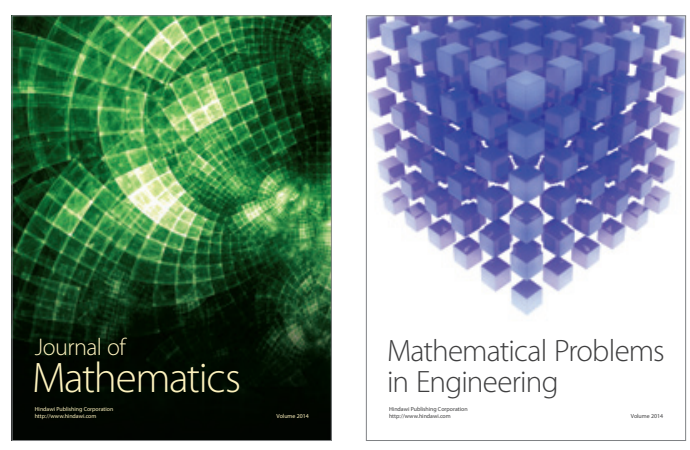

Mathematical Problems in Engineering
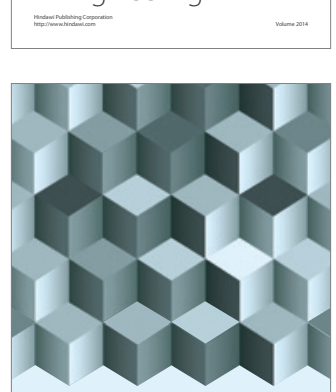

Journal of

Function Spaces
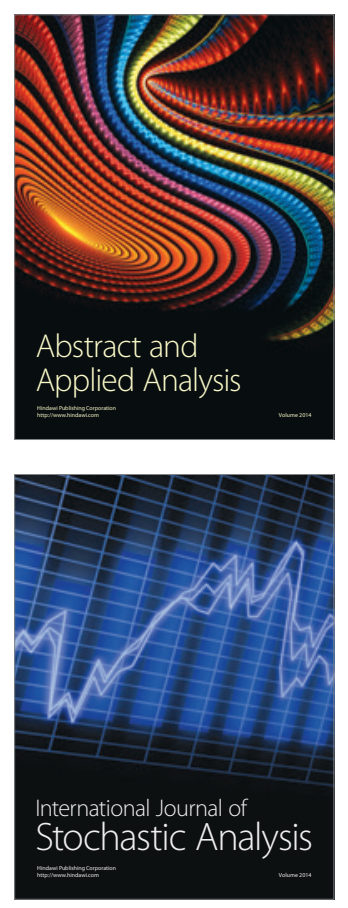

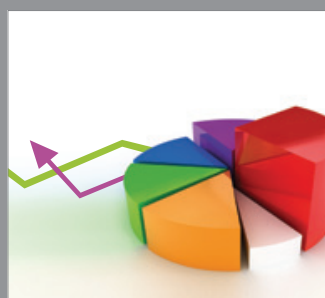

ournal of

Probability and Statistics

Promensencen
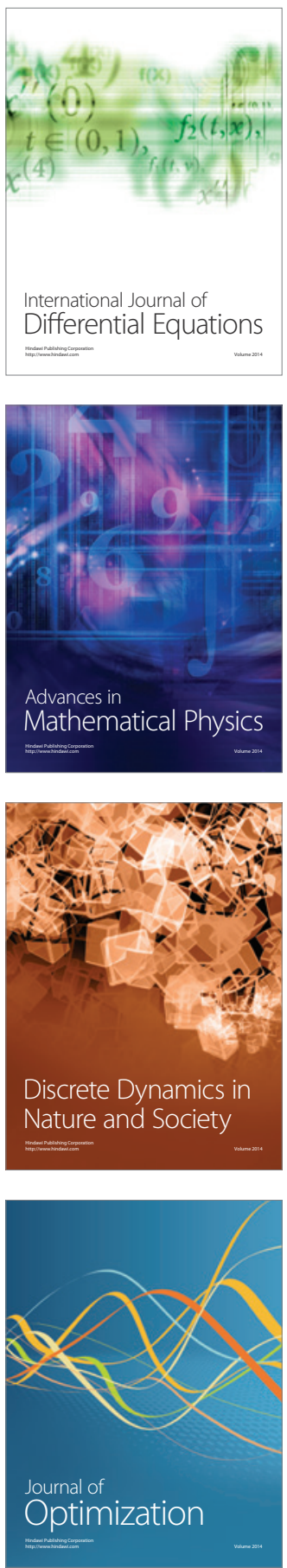